\title{
Large scale magnetic fields and coherent structures in nonuniform unmagnetized plasma
}

\author{
Martin Jucker \\ École Polytechnique Fédérale de Lausanne, CH-1015 Lausanne, Switzerland \\ Zhanna N. Andrushchenko \\ Swedish University of Agricultural Sciences, SE-750 07 Uppsala, Sweden \\ Vladimir P. Pavlenko ${ }^{\text {a) }}$ \\ Uppsala University and EURATOM-VR Fusion Association, SE-751 20 Uppsala, Sweden
}

(Received 5 January 2006; accepted 26 June 2006; published online 28 July 2006)

\begin{abstract}
The properties of streamers and zonal magnetic structures in magnetic electron drift mode turbulence are investigated. The stability of such large scale structures is investigated in the kinetic and the hydrodynamic regime, for which an instability criterion similar to the Lighthill criterion for modulational instability is found. Furthermore, these large scale flows can undergo further nonlinear evolution after initial linear growth, which can lead to the formation of long-lived coherent structures consisting of self-bound wave packets between the surfaces of two different flow velocities with an expected modification of the anomalous electron transport properties.
\end{abstract}

(C) 2006 American Institute of Physics. [DOI: 10.1063/1.2234289]

\section{INTRODUCTION}

The generation of large scale flows by underlying small scale turbulence is a well known phenomenon in different areas of current research. Some examples are the Rossby waves and the jet stream in atmospheric physics, the tidal currents in the seas or the Jovian belt, and the rotation of Venus' atmosphere in astrophysics. In plasma physics, the generation of such flows, defined as large scale length nonlinear structures with additional symmetry, so-called zonal flows and streamers, by drift wave turbulence has been widely investigated because of their critical role in anomalous transport, particularly in fusion devices. ${ }^{1}$ Zonal flows correspond to elongated structures perpendicular to the direction of plasma inhomogeneity, whereas streamers are elongated along that direction. Growth of such highly anisotropic secondary flows is commonly attributed to the effect of Reynolds stress. ${ }^{2}$ Once they are excited, they form an environment for the parent drift-type waves. Whereas the short waves must ride on the longer structures and be modulated by them, the latter exist in a background of short wavelength turbulence which can exert significant stress on the structures. Since the total wave energy is conserved, the parent waves and the secondary flows form therefore a selfregulating system and cannot be addressed in isolation. ${ }^{3}$

An advanced approach to describe this self-regulation is to present drift waves and flow spectra as coupled elements of the self-organized turbulence. Then the coupling of slow large scale perturbations and modulations of fast small scale background drift turbulence can conveniently be described by the wave kinetic equation for a generalized wave action invariant. ${ }^{4,5}$ For the electrostatic drift wave turbulence, such a spectral model addressed from the perspective of flow generation and self-regulation by feedback of zonal flow on the

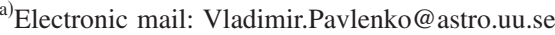

drift wave spectrum has recently been developed. ${ }^{3,6,7}$ The model consists of two evolution equations. One of them is the equation for the zonal flow amplitude, which is driven by modulationally unstable drift waves. So, the flow generation is considered in the context of a random phase approximation modulational instability, which is appropriate for fully developed wave turbulence. The second is the evolution equation that governs the dynamics of drift waves in the straining field of zonal flows. Different regimes of zonal flow generation by drift waves, the back-reaction of the flows on the drift wave turbulence via random shearing as well as various feedback loops by which the system regulates itself have been investigated in the frame of this model. ${ }^{1,8-10}$

In this paper, we turn our attention to the magnetic electron drift mode turbulence. The corresponding turbulent fluctuations are drift-type modes excited in a nonuniform unmagnetized plasma, characterized by a frequency range in between the electron and the ion plasma frequencies. Our interest in this particular regime is motivated by earlier studies of these modes, which suggest a possibility of spontaneous generation of strong magnetic fields in laser produced plasmas, which have been observed since the 1970s. Moreover, phenomena occurring in such time scales may even be more important as a source of the secondary magnetic field structures and are often encountered in space physics, ${ }^{11,12}$ as well as in a number of plasma operated devices (e.g., switches, focusing devices, $\mathrm{Z}$ pinches, etc.). ${ }^{13}$ The linear theory of the modes, developed on the basis of fluid models, ${ }^{14-18}$ shows that unstable motion is fed by inhomogeneities in the equilibrium electron density and temperature. The long wavelength limit $\left(k \ll \omega_{p i} / c\right.$, where $\omega_{p i}$ is the ion plasma frequency) is usually described by neglecting the electron inertia with ${ }^{15}$ or without ${ }^{14}$ a hydrodynamical response of the plasma. In the opposite limit, $k \gg \omega_{p i} / c$, the ion fluid velocity is negligible and electron dynamics must be 
included. This inclusion has been discussed and accepted in a number of papers. ${ }^{16-20}$ We will restrict ourselves to this limit. In this case, the source of the instability is the baroclinic vector $\nabla n_{0} \times \nabla T_{e}$ in the electron fluid $\left(n_{0}\right.$ is the background density and $T_{e}$ the electron temperature), which gives rise to a finite vorticity. The mode does not depend on collisional effects or heat flux. ${ }^{16,18}$ The electron inertia, which manifests itself in the electron fluid vorticity, and the temperature perturbation are then essential for these modes.

The linear instability can explain the observed strong magnetic fields excited in unmagnetized plasma in the frequency range measured. But, once the magnetic field reaches a certain level, nonlinear interactions become important and the linear description is not valid any longer. Furthermore, even in the stable region we will consider here, linear theory is not capable of describing large scale structures, which we are expecting to appear. Therefore the nonlinear theory for magnetic electron drift modes has been developed in a series of papers. ${ }^{21-26}$ The development of this theory results in a set of two-field nonlinear model equations, contrary to the theory of electrostatic drift waves, which can be cast in terms of magnetic field and electron temperature. ${ }^{21}$ There has been considerable interest lately to understand the nonlinear features of the magnetic electron drift mode. There, the Hamiltonian structure and integrals of motion, the highly localized dipolar and monopolar magnetic vortices, as well as magnetic vortex streets, were identified and the stability of these solutions was investigated. The statistical properties of twodimensional magnetic electron drift turbulence in terms of power spectra and cascade properties of square invariants supported by the model were outlined and a double energy cascade was demonstrated to exist due to the presence of two invariants. ${ }^{27}$ On the basis of the existence of these quadratic invariants it can be inferred that the mean square magnetic field cascades towards longer scales.

Within the magnetic electron drift mode turbulence, we can address the question of generation of large scale magnetic fields by small scale turbulent magnetic fluctuations in a way similar to the flow generation by electrostatic drift turbulence. ${ }^{1}$ Since for the magnetic electron drift mode turbulence we assume equilibrium density and temperature gradients, we can expect that a significant portion of the free energy stored in these gradients will be converted to large scale fields via magnetic Reynolds stress and thus the excitation of secondary highly symmetric large scale magnetic fields in our two-field model as well. ${ }^{25}$ However, these large scale structures are not flows in the original sense, since it is not the flow of the particles, but rather magnetic structures which are elongated along one direction and periodical with a long wavelength along the other direction as well. Following this similarity we will distinguish the two corresponding limits "zonal magnetic fields" (ZF) and "magnetic streamers" (MS), as it has been adopted in the literature. ${ }^{1,11,13,28-31}$ Despite many similarities between the flows and large scale magnetic fields, the extension is not trivial. Indeed, as a first point, magnetic electron drift mode turbulence is a two-field model. This model depicts phenomena having a scale length shorter than the ion skin depth. For such phenomena, the evolution of the magnetic field is governed by the electron flow velocity. The ions being stationary, the flow velocity of the electrons determines the current and hence it is directly related to the curl of the instantaneous magnetic field. In this approximation, the magnetic field itself evolves through an explicitly nonlinear equation. ${ }^{21}$ The second distinction arises in the presence of an intrinsic scale, the electron skin depth $\lambda=c / \omega_{p e}$, in the magnetic drift turbulence model, which separates the two regimes: One in which the electron inertia is important, and the other where it plays no role. The character of the evolution equations changes in these two disparate regimes of scale lengths.

Turning to the methodology of the magnetic electron drift wave-large scale structure self-regulation, we note that the total wave energy is conserved and contains both the part stored in small as well as large scale structures. We thus deal with a coupled system of two different parts of the same wave spectrum, which cannot be addressed in isolation. This necessitates a theory capable of describing magnetic electron drift wave and large scale magnetic field spectra as interacting parts of magnetic electron drift mode turbulence, similar to that developed for the electrostatic drift turbulence. ${ }^{3}$ Recently, such a spectral model for magnetic electron drift mode turbulence has been developed. ${ }^{26}$ This model includes not only the generation of large scale magnetic fields by underlying microturbulence, but also the back reaction of these fields on the small scale magnetic drift mode turbulence via the wave kinetic equation for a suitable action-like invariant.

Having this spectral model equation in our hand, we focus in the present paper on the dynamics of interacting magnetic drift wave-large scale field turbulence. First, different regimes (kinetic and hydrodynamic) of the generation of large scale magnetic fields, zonal magnetic fields, and magnetic streamers, will be investigated in detail and the conditions for such excitation to happen will be stressed out. Having thus shown that there are mechanisms assuring longlived large scale magnetic fields, we study then how these fields can evolve even further in a nonlinear way. We will show that one possible nonlinear solution is corresponding to a coherent solitary structure, which can be created in between layers of different magnetic flow velocity. It is worthwhile to point out that in these studies the large scale magnetic fields cannot be considered as independent "species" in the complete spectrum, since they are always interacting with the underlying turbulence and by means of the wave kinetic equation this interaction is always taken into account. The formation of such coherent structures can be of interest in the context of an electron transport mechanism, which has a smaller characteristic scale and a larger growth rate than the ion turbulence. ${ }^{29}$

These long-lived coherent structures may change the properties of the electron transport in that e.g. a strong impermeability is formed between the inside and outside of such a structure, associated with long-term advection of trapped particles. Moreover, these coherent structures can propagate perpendicular to the elongation and thus favor anomalous transport in that direction. This effect is exactly opposite to the transport inhibiting property of large scale flows via shearing of small scale turbulence. 
The rest of the paper is organized as follows: In Sec. II we will recall the model equations for magnetic electron drift mode turbulence and its limits for zonal magnetic fields (ZF) and magnetic streamers (MS) as well as the wave kinetic equation and its action-like invariant. In Sec. III, two different regimes of large scale magnetic field excitation are investigated, and Sec. IV deals with the evolution equation for ZF and MS. In Sec. V a stationary solution describing coherent structures of this evolution equation is presented. Finally, the article is completed with a conclusion and discussion in Sec. VI.

\section{BASIC EQUATIONS}

Magnetic electron drift modes are drift-type motions of a nonuniform unmagnetized plasma with typical frequencies of the order of $\kappa v_{T}$, where $\kappa$ is the inverse characteristic length of the background inhomogeneity and $v_{T_{e}}$ is the thermal electron velocity. To describe these modes, we consider a nonuniform unmagnetized plasma with an equilibrium density $n_{0}$ and an equilibrium electron temperature $T_{0}$. The time scale of interest is in between the inverse ion and the inverse electron plasma frequency, and hence we consider an unpolarized electron fluid and immobile ions. The massive ions play a passive role as a neutralizing background, and the dominant role in dynamics is played by electron species.

In order to derive the model equations describing the nonlinear dynamics of the magnetic electron drift mode, the momentum equation together with Maxwell's equations and the energy equation are used. ${ }^{21}$ We make the standard assumptions that we consider a two-dimensional case in the $x$ $y$ plane. Then, all quantities are independent of $z$ and the perturbed magnetic field is directed along the $z$ axis. The length scales of the fluctuations are supposed to be much smaller than those of the equilibrium quantities. The temperature will be considered the sum of an equilibrium and a perturbed part $T_{0}+T$ and the perturbed magnetic field is denoted by $B$. As a last assumption we consider the equilibrium density and temperature $n_{0}$ and $T_{0}$ as functions of $x$ only. Taking the curl of the momentum equation one can show, with all the above assumptions, that the basic system of equations describing both linear and nonlinear properties of magnetic electron drift modes becomes ${ }^{21}$

$$
\begin{aligned}
& \frac{\partial T}{\partial t}+\alpha \frac{\partial B}{\partial y}=-\frac{e \lambda^{2}}{m}\{B, T\}, \\
& \frac{\partial}{\partial t}\left(B-\lambda^{2} \nabla^{2} B\right)+\beta \frac{\partial T}{\partial y}=\frac{e \lambda^{4}}{m}\left\{B, \nabla^{2} B\right\} .
\end{aligned}
$$

Here $\alpha=\lambda^{2}\left(e T_{0} / m\right)\left(3 / 2 \kappa_{n}-\kappa_{T}\right), \beta=\kappa_{n} / e, \quad \kappa_{n}=|\nabla \ln n|$ and $\kappa_{T}=\left|\nabla \ln T_{0}\right|$ are the inverse length scales of the density and the temperature inhomogeneities and $\lambda$ is the skin depth.

Note that the evolution equation for the magnetic field is nonlinear in $B$. This is intrinsically due to the convective derivative in the electron momentum equation. The order of perturbation of the RHS in (1a) (representing the baroclinic vorticity source) shows that the perturbed temperature should not be neglected.
Linearizing the evolution equations (1) for small perturbations $B, T \propto \exp (-i \omega t+i \mathbf{k r})$, the dispersion relation for magnetic electron drift modes is obtained with the linear wave eigenfrequency ${ }^{18,19}$

$$
\omega_{k}=k_{y} \sqrt{\frac{\alpha \beta}{1+k^{2} \lambda^{2}}},
$$

where $\alpha \beta=v_{t h}^{2} \lambda^{2} \kappa_{n}\left(2 / 3 \kappa_{n}-\kappa_{T}\right)$. There is a purely growing solution for $\kappa_{T}>(2 / 3) \kappa_{n}$, which can explain the measured strong magnetic fields in laser fusion. However, as discussed in the Introduction, linear theory is not of great use in our case. In fact, we will consider $\omega_{k}$ to be real in this article, i.e., $\alpha \beta>0$. Another useful result from the Fourier decomposition in the model equations is that one can find a relation between the magnetic field Fourier amplitudes $B_{k}$ and those of the temperature $T_{k}$, which is

$$
T_{k}=\frac{\alpha k_{y}}{\omega_{k}} B_{k} .
$$

We have seen that the underlying magnetic electron drift mode turbulence is driven by gradients of temperature and density. As already mentioned, this microturbulence can spontaneously generate large scale magnetic flows ${ }^{25}$ via fluctuation-induced magnetic Reynolds stress. For a description of the system large scale fields (microturbulence), we have to consider nonlinear effects, since the linear regime cannot explain the appearance of large scale structures, and we use the ansatz of multiple scale expansion between the spatio-temporal scales of the fields and those of the microturbulence. We thus assume a sufficient spectral gap separating large and small scale motions. ${ }^{1}$ The temperature and the magnetic field are then decomposed into a large scale, slowly varying (denoted with a bar) and a small scale component, $\bar{T}+\widetilde{T}$ and $\bar{B}+\widetilde{B}$. Having the nonlinear model equation in our hands, we are then able to describe the interaction between small and large scale structures within the turbulence. The separation of the whole turbulent spectrum into two parts, one describing large scale structures with a wave vector denoted by $\mathbf{q}$, and another one describing small scale turbulence, whose wave vector is denoted by $\mathbf{k}$ is mathematically described by

$$
\begin{aligned}
\left(\begin{array}{c}
B \\
T
\end{array}\right)(\mathbf{r}, t)= & \sum_{k}\left(\begin{array}{c}
B_{k} \\
T_{k}
\end{array}\right)(t) e^{i \mathbf{k r} \quad \text { (small scales) }} \\
& +\left(\begin{array}{c}
B_{q} \\
T_{q}
\end{array}\right)(t) e^{i \mathbf{q r}}+\text { c.c. (large scales), }
\end{aligned}
$$

where obviously $|\mathbf{k}| \gg|\mathbf{q}|$. This description is inserted into our model equations (1) and neglecting the noise emitted into the fields by incoherent coupling of magnetic electron drift modes, we find the evolution equations for large scale fields. In the limit of zonal magnetic fields, $\mathbf{q}=(q, 0,0)$, the above decomposition (4) yields together with the model equations (1)

$$
\frac{\partial T_{q}}{\partial t}=0
$$




$$
\frac{\partial B_{q}}{\partial t}=\frac{e \lambda^{2}}{m} \frac{q^{2} \lambda^{2}}{1+q^{2} \lambda^{2}} \int d^{2} \mathbf{k} k_{x} k_{y}\left|B_{k}\right|^{2} .
$$

For magnetic streamers, $\mathbf{q}=(0, q, 0)$, Eqs. (1) become

$$
\begin{aligned}
& \frac{\partial T_{q}}{\partial t}+i \alpha q B_{q}=0, \\
& \frac{\partial B_{q}}{\partial t}+i \beta \frac{q}{1+q^{2} \lambda^{2}} T_{q}=-\frac{e \lambda^{2}}{m} \frac{q^{2} \lambda^{2}}{1+q^{2} \lambda^{2}} \int d^{2} \mathbf{k} k_{x} k_{y}\left|B_{k}\right|^{2} .
\end{aligned}
$$

It is a direct consequence from these equations that the mean electron temperature associated with zonal fields does not evolve with time. A clear difference between the evolution of magnetic streamers (MS) and zonal fields (ZF) can immediately be stated. Whereas the evolution equations of $\mathrm{ZF}$ are decoupled and the temperature can even be considered as a constant, the equations for MS remain coupled and they always have a real frequency in contrast to zero-frequency ZF. MS have a linear eigenfrequency and therefore linear instability is possible in this case. There is, however, an important similarity between the two large scale structures, in that they are excited nonlinearly by the same small scale turbulence via the magnetic Reynolds stress $\sum_{k} k_{x} k_{y} B_{k} B_{-k}$. This is of course not unexpected, since the nonlinear excitation of large scale flows by Reynolds stress already is well established for electrostatic drift wave turbulence. ${ }^{1,2}$

Once we have found the excitation of large scale fields by small scale turbulence, we have to determine the "loop back," i.e., the response of the large scale structures on the small scales for describing the nonlinear evolution of the total wave spectrum in a self-consistent way. In order to do so, it is appropriate to consider the evolution of this microturbulence in a medium which is slowly modulated by large scale structures. This can conveniently be described by the help of a wave kinetic equation for the wave action density $N_{k}(\mathbf{r}, t)$ in $\mathbf{r}-\mathbf{k}$ space. ${ }^{4}$ Since the standard expression for the wave action, $N_{k}=E / \omega_{k}$ (where $E$ is the wave energy), can only be used when the total energy is contained in the small scale waves, it is not suitable for a system with mean flow, ${ }^{5,32}$ where the total energy containing small and large scale quantities is conserved. However, the appropriate wave action invariant has recently been derived ${ }^{26}$ and takes the form

$$
\left|N_{k}\right|=\left|\psi_{k}\right|^{2}=4 \frac{\alpha}{\beta}\left(1+k^{2} \lambda^{2}\right)\left|B_{k}\right|^{2} .
$$

The corresponding wave kinetic equation can then be written as

$$
\frac{\partial N_{k}}{\partial t}+\frac{\partial \omega_{k}^{N L}}{\partial \mathbf{k}} \frac{\partial N_{k}}{\partial \mathbf{r}}-\frac{\partial \omega_{k}^{N L}}{\partial \mathbf{r}} \frac{\partial N_{k}}{\partial \mathbf{k}}=2 \gamma_{k} N_{k}-\operatorname{St}\left(N_{k}\right) .
$$

The terms on the RHS account for wave damping due to linear and nonlinear mechanisms, as well as local wave interactions, and guarantee saturation in the absence of large scale fields. The form of $S t\left(N_{k}\right)$ is not important here. Note that the linear frequency of the magnetic electron modes (2) is assumed to be real and the proper way to introduce a possible linear instability of the small scale turbulence is through a linear instability of $N_{k}$, i.e., the first term on the RHS. ${ }^{4}$

The linear frequency is modified in the presence of large scale fields. The reason is the Doppler shift induced by the (large scale) "flow velocity." Therefore the nonlinear frequency $\omega_{k}^{\mathrm{NL}} \equiv \omega_{k}^{\mathrm{Re}}+\Delta$ has been introduced. Explicitly, $\Delta$ can be written as

$$
\Delta=\frac{1+2 k^{2} \lambda^{2}}{1+k^{2} \lambda^{2}} \mathbf{k} \cdot \mathbf{v}_{B}^{(q)}-\frac{1}{\sqrt{1+k^{2} \lambda^{2}}} \mathbf{k} \cdot \mathbf{v}_{T}^{(q)},
$$

where we introduced the large scale velocities depending on the magnetic field and the temperature gradients

$$
\mathbf{v}_{B} \equiv-\frac{e \lambda^{2}}{4 m}(\nabla B \times \hat{\mathbf{z}})
$$

and

$$
\mathbf{v}_{T} \equiv-\frac{e \lambda^{2}}{4 m} \sqrt{\frac{\beta}{\alpha}}(\nabla T \times \hat{\mathbf{z}}) .
$$

It is convenient for the rest of this paper to introduce the "magnetic flow velocity"

$$
\mathbf{v}_{f} \equiv \frac{1+2 k^{2} \lambda^{2}}{1+k^{2} \lambda^{2}} \mathbf{v}_{B}^{(q)}-\frac{1}{\sqrt{1+k^{2} \lambda^{2}}} \mathbf{v}_{T}^{(q)},
$$

where the superscript $(q)$ means that only the large scale spectral component of the magnetic field and the temperature have to be considered in (10). With this definition, we can write $\Delta=\mathbf{k} \cdot \mathbf{v}_{f}$. Note that the picture of the flow velocity comes from the analogy to the electrostatic drift wave turbulence, where an actual flow of particles is described. However, in addition to the mathematical similarities, there are two points which strengthen this analogy: First, in the very low frequency limit where the MHD description is valid $\left(\omega \ll \omega_{p i}\right)$, magnetic field lines are "frozen" into the plasma, ${ }^{13}$ i.e., particles and magnetic field move together. So, there is a certain tradition of connecting the motion of the particles with the motion of the magnetic field. However, it is very important to note here that these approximations are not valid in this article, since we are dealing with much higher frequencies $\left(\omega \gg \omega_{p i}\right)$. The second point is that for magnetic electron drift mode turbulence, the first order drift velocity of the particles is $\mathbf{v}_{D} \sim \nabla B \times \hat{\mathbf{z}},{ }^{21}$ and it is perpendicular to the $z$ axis and the gradient of the magnetic field. If we now have, for instance, a zonal magnetic field, the gradient is along the $x$ axis and thus the particle flow is along the $y$ axis, which is the direction of elongation of the large scale magnetic field and the direction of the flow velocity $v_{f}$ as well. In fact, the part of the flow velocity $v_{f}$ due to the magnetic field (10a) is exactly one fourth of the particle drift velocity $\mathbf{v}_{D} \cdot{ }^{26}$ We note in this context, since the amplitude of the magnetic field can be rather high, the gradient (the field oscillates between $-\left|B_{q}\right|$ and $\left.\left|B_{q}\right|\right)$ generating the particle drift may be high as well. In that sense, there is a certain relation between the instantaneous magnetic field and the actual particle flow. So, the "flow velocity" determined by (11) is not the velocity of some particles within the plasma, but nevertheless, due to the 
similarity discussed above, we will keep this definition for the magnetic flow velocity $v_{f}$.

The wave kinetic equation (8) describes the evolution of the small scale wave spectrum in a medium with slowly varying parameters. In other words, it describes in a selfconsistent way the evolution of the envelope of small scale turbulence due to interaction with large scale fields generated by the very turbulence itself. Decomposing the wave spectrum into an equilibrium and a perturbed part, $N_{k}=N_{0}+\tilde{N}_{k}$, and assuming that the equilibrium spectrum evolves slowly with time and over large spatial scales, one can average the wave kinetic equation over the fast/short scales, and obtain the equation for the equilibrium distribution, ${ }^{4}$

$$
\frac{\partial N_{0}}{\partial t}-\overline{\frac{\partial}{\partial \mathbf{r}}\left(\mathbf{k} \cdot \mathbf{v}_{f}\right) \frac{\partial \widetilde{N}}{\partial \mathbf{k}}}=0 .
$$

The equation for the perturbed part can then be found by subtracting (12) from (8)

$$
\frac{\partial \tilde{N}}{\partial t}+\mathbf{v}_{g} \frac{\partial \tilde{N}}{\partial \mathbf{r}}-\frac{\partial}{\partial \mathbf{r}}\left(\mathbf{k} \cdot \mathbf{v}_{f}\right) \frac{\partial N_{0}}{\partial \mathbf{k}}=0 .
$$

Then, if we assume $\tilde{N}_{k} \sim \exp (-i \Omega t+i \mathbf{p} \cdot \mathbf{r})$, the solution of Eq. (13) can be written as

$$
\tilde{N}=\frac{\partial}{\partial \mathbf{r}}\left(\mathbf{k} \cdot \mathbf{v}_{f}\right) \frac{\partial N_{0}}{\partial \mathbf{k}} R(\Omega, p),
$$

where the response function $R(\Omega, p)=i /\left(\Omega-p v_{g}\right)$ was introduced. The obtained results will be used in the succeeding sections investigating different regimes of large scale field generation.

\section{EXCITATION OF LARGE SCALE FIELDS}

In the previous section, it was stressed out that large scale magnetic fields can be generated by small scale turbulence via magnetic Reynolds stress, as might be seen from Eqs. (5) and (6). However, such information is of very general nature and does not give us more detailed knowledge on how and when such large scale fields are generated by underlying turbulence. It is indeed only a necessary condition for the generation and does not permit us to determine neither a sufficient condition for the excitation nor the increment. The aim of the present section is to point out these conditions by investigating different regimes of large scale field generation, using the self-consistent model of Eqs. (5)-(8), and to find the corresponding rate of excitation.

\section{A. Kinetic regime}

In our first approach we are looking for a general criterion for the generation of large scale fields, depending on the form of the wave spectrum.

In the case of zonal magnetic fields, the basis of our investigation are Eqs. (5). Introducing the large scale "vector"

$$
\bar{B} \equiv \frac{e \lambda^{2}}{4 m}\left(B_{q}-\sqrt{\frac{\beta}{\alpha}} T_{q}\right) e^{i \mathbf{q} \cdot \mathbf{r}}
$$

we get from the model equations, taking their difference, the equation of motion for $\bar{B}$,

$$
\frac{\partial \bar{B}}{\partial t}=\frac{1}{4}\left(\frac{e \lambda^{2}}{m}\right)^{2} \frac{q^{2} \lambda^{2}}{1+q^{2} \lambda^{2}} \int\left|B_{k}\right|^{2} k_{x} k_{y} d^{2} \mathbf{k} .
$$

For simplicity, let us introduce the coefficient $K_{q}$ defined by

$$
K_{q}^{2}:=\frac{\beta}{16 \alpha} \frac{e^{2} \lambda^{2}}{m^{2}} \frac{q^{2} \lambda^{2}}{1+q^{2} \lambda^{2}} .
$$

As a first step, we will use (7) in order to replace the magnetic field Fourier components in the integral of (16) with the wave spectrum. Then we decompose $N_{k}$ into an equilibrium and a perturbed part, $N_{k}=N_{0}+\widetilde{N}_{k}$, and use (14), in order to replace the perturbed wave spectrum with the corresponding expression proportional to $\bar{B}$. Now let $\partial / \partial t \sim-i \Omega^{z f}+\gamma^{z f}$, so that (16) finally becomes

$$
\gamma^{z f}-i \Omega^{z f}=-K_{q}^{2} \int q_{x}^{2} \frac{k_{y}^{2} \lambda^{2}}{1+k^{2} \lambda^{2}} k_{x} \frac{\partial N_{0}}{\partial k_{x}} R(\Omega, p) d^{2} \mathbf{k} .
$$

The problem is similar to the one described in Refs. 33 and 34 , where the instability of a gas of plasmons (described by $N_{k}$ ) due to coupling with ion-acoustic waves is described. The response function is then $R(\Omega, p)=i /\left(\Omega-p v_{g}+i \gamma^{N}\right)$, where $\gamma^{N}$ is small and positive. The strongest interaction between the small scale turbulence (the medium), presented by its wave spectrum $N_{0}$, and the zonal fields can be expected when the reaction of the medium $\left(\Omega^{N}\right.$ in the response function) is in resonance with the perturbation (represented by $v_{g}$ in the response function), that is, when $\Omega^{N} \simeq \mathbf{p} \cdot \mathbf{v}_{g}$ and thus $R(\Omega, p) \sim 1 / \gamma^{N}>0$. Because of the strong interaction, we can assume $\Omega^{z f} \simeq 0$. The corresponding regime of flow generation is called "kinetic" because of its obvious similarities with Landau damping in kinetic wave theory. The result of these considerations is the criterion for instability

$$
\gamma^{z f}>0 \Leftrightarrow k_{x} \frac{\partial N_{0}}{\partial k_{x}}<0 .
$$

This result is the opposite of the condition for the Langmuir turbulence, where the slope of the velocity distribution function must be positive for positive velocities.

If the $\mathrm{ZF}$ amplitude grows due to the above instability, the nonesonant response becomes important as well. Thus, we investigate the nonresonant part $\Omega^{\mathrm{zf}}$ assuming $\Omega^{N} \ll \mathbf{p} \cdot \mathbf{v}_{g}$ and $\gamma^{2 \mathrm{f}} \simeq 0$. In this limit, we can use the same Eq. (18), but with the expression for the response function now being $R(\Omega, p) \sim-i / q v_{g}$ and thus, as for the resonant part,

$$
\Omega^{z f}>0 \Leftrightarrow k_{x} \frac{\partial N_{0}}{\partial k_{x}}<0
$$

with 


$$
\Omega^{z f}=-K_{q}^{2} \int q_{x}^{2} \frac{k_{y}^{2} \lambda^{2}}{1+k^{2} \lambda^{2}} k_{x} \frac{\partial N_{0}}{\partial k_{x}} \frac{1}{q_{x} v_{g}} d^{2} \mathbf{k} .
$$

With a similar approach as for zonal fields, but using Eqs. (6), the case of magnetic streamers can be studied. In this case, the equivalent to (16) is

$$
\frac{\partial \bar{B}}{\partial t}-i \omega_{q} \bar{B}=\frac{1}{4}\left(\frac{e \lambda^{2}}{m}\right)^{2} \frac{q^{2} \lambda^{2}}{1+q^{2} \lambda^{2}} \int\left|B_{k}\right|^{2} k_{x} k_{y} d \mathbf{k},
$$

where we defined $\omega_{q} \equiv q \sqrt{\alpha \beta}$. Taking $\partial / \partial t \sim-i \Omega^{s t t}+\gamma^{s t}$ and redefining $\Omega^{s t}=\Omega^{s t}+\omega_{q}$, one obtains analogously to the above case the criterion for instability

$$
\gamma^{s t}>0 \Leftrightarrow k_{y} \frac{\partial N_{0}}{\partial k_{y}}>0,
$$

and in the nonresonant limit the frequency

$$
\Omega^{s t}>0 \Leftrightarrow k_{y} \frac{\partial N_{0}}{\partial k_{y}}>0,
$$

where

$$
\Omega^{s t}=K_{q}^{2} \int q_{y}^{2} \frac{k_{x}^{2} \lambda^{2}}{1+k^{2} \lambda^{2}} k_{y} \frac{\partial N_{0}}{\partial k_{y}} \frac{1}{q_{y} v_{g}} d^{2} \mathbf{k} .
$$

Note that the conditions (23) and (24) are exactly contrary to zonal fields and similar to Langmuir turbulence.

\section{B. Hydrodynamic regime}

In the previous section, we derived a rather general criterion for excitation of large scale magnetic fields by small scale turbulence, which depends on the equilibrium spectrum distribution. However, in order to have an exact result in the case of zonal magnetic fields, one has to integrate Eq. (16) or, which comes to the same after replacing the magnetic field Fourier amplitudes as before, integrate

$$
-i \Omega^{z f}=-K_{q}^{2} q^{2} \int \frac{k_{y}^{2} \lambda^{2}}{1+k^{2} \lambda^{2}} k_{x} \frac{\partial N_{0}}{\partial k_{x}} R(\Omega, p) d^{2} \mathbf{k} .
$$

For explicit integration, one has to consider a specific form of the equilibrium wave spectrum $N_{0}$. We will consider a monochromatic spectrum which can easily be integrated. The resulting dispersion relation will be of a hydrodynamic type, since through integration all harmonics have been considered. Before starting calculations, it is worthwhile to note that in what follows, $\Omega^{\mathrm{zf}} \approx \Omega$ and $q^{\mathrm{zf}} \approx p$, since we are considering a modulation of the magnetic drift wave turbulence by the large scale field. The response function comes from the wave kinetic equation and is due to the term $\partial \omega / \partial \mathbf{k} \cdot \partial N_{k} / \partial \mathbf{x}$, which takes in the case of zonal fields the form $\partial \omega / \partial \mathbf{k} \cdot \hat{\mathbf{x}} q_{x} N_{k}$, and therefore $v_{g}=\frac{\partial \omega}{\partial k_{x}}$. The frequency $\omega$ is a sum of the linear frequency and a nonlinear effect due to mean "flow," corresponding to a simple Doppler shift. Therefore, we only take into account the linear frequency defined in (2) for the group velocity. Explicitly, the group velocity of the small scale perturbation is

$$
v_{g}=-\sqrt{\alpha \beta} \frac{k_{x} k_{y} \lambda^{2}}{\left(1+k^{2} \lambda^{2}\right)^{3 / 2}} .
$$

Now we proceed with the investigation of Eq. (26). Assuming a monochromatic wave packet $N_{0}^{k}=N_{0} \delta\left(\mathbf{k}-\mathbf{k}_{\mathbf{0}}\right)$, and performing the integration by parts yields the dispersion relation for $\mathrm{ZF}$

$$
\begin{aligned}
\Omega= & -K_{q}^{2} q^{2} \int k_{y}^{2} \lambda^{2} N_{0} \delta\left(\mathbf{k}-\mathbf{k}_{0}\right) \frac{\partial}{\partial k_{x}} \\
& \times\left(\frac{1}{\Omega-q v_{g}} \frac{k_{x}}{1+k^{2} \lambda^{2}}\right) d^{2} \mathbf{k} .
\end{aligned}
$$

Taking into account (27), we can replace the factor in the brackets with a term depending on the group velocity:

$$
\frac{k_{x}}{1+k^{2} \lambda^{2}}=-\frac{v_{g}}{\sqrt{\alpha \beta} k_{y} \lambda^{2} \sqrt{1+k^{2} \lambda^{2}}} .
$$

In the limit $k \lambda \ll 1$, the replacement leads to the term

$$
\begin{aligned}
\frac{\partial}{\partial k_{x}}\left(\frac{1}{\Omega-q v_{g}} \frac{k_{x}}{1+k^{2} \lambda^{2}}\right) & =-\frac{1}{\sqrt{\alpha \beta} k_{y} \lambda^{2}} \frac{\partial}{\partial k_{x}}\left(\frac{v_{g}}{\Omega-q v_{g}}\right) \\
& =-\frac{1}{\sqrt{\alpha \beta} k_{y} \lambda^{2}} \frac{\Omega}{\left(\Omega-q v_{g}\right)^{2}} \frac{\partial v_{g}}{\partial k_{x}},
\end{aligned}
$$

and thus, the integration of the dispersion relation (28) is trivial with the result

$$
\left(\Omega-q v_{g}\right)^{2}=\left.K_{q}^{2} q^{2} k_{0 y}^{2} \frac{N_{0}}{\sqrt{\alpha \beta} k_{0 y}} \frac{\partial v_{g}}{\partial k_{x}}\right|_{k_{0}} .
$$

We see directly from the last equation that the requirement for instability is

$$
\left.\frac{N_{0}}{\sqrt{\alpha \beta} k_{0 y}} \frac{\partial v_{g}}{\partial k_{x}}\right|_{k_{0}}<0 .
$$

Explicit calculations of the derivative of the group velocity yields the exact expression for the large scale zonal structure's complex frequency

$$
\Omega=q v_{g}-i K_{q}|q| \frac{\left|k_{0 y} \lambda\right|}{\left(1+k^{2} \lambda^{2}\right)^{5 / 2}} N_{0}^{1 / 2} \sqrt{1-2 k_{0 x}^{2} \lambda^{2}+k_{0 y}^{2} \lambda^{2}},
$$

with the imaginary part being the rate of generation of large scale fields. Note that stabilization takes place for $1-2 k_{0 x}^{2} \lambda^{2}+k_{0 y}^{2} \lambda^{2}<0$, corresponding to a negative derivative of the group velocity.

In the opposite limit, $k \lambda \gg 1$, the dispersion relation looks like

$$
1=K_{q}^{2} q^{2} \int d^{2} \mathbf{k} k_{y}^{2} N_{0}^{k} \frac{1}{k_{y} \sqrt{\alpha \beta}} \frac{1}{k \lambda} \frac{1}{\left(\Omega-q v_{g}\right)^{2}} \frac{\partial v_{g}}{\partial k_{x}} .
$$

Assuming a monochromatic distribution and integrating yields then 


$$
\left(\Omega-q v_{g}\right)^{2}=\left.K_{q}^{2} q^{2} k_{0 y}^{2} \frac{N_{0}}{\sqrt{\alpha \beta} k_{0 y}} \frac{1}{k_{0} \lambda} \frac{\partial v_{g}}{\partial k_{x}}\right|_{k_{0}} .
$$

Note that this is exactly the same expression as in the case $k \lambda \ll 1$, (30), but the instability is reduced by a factor $1 / k \lambda \ll 1$. As before, there is instability for

$$
\left.\frac{N_{0}}{\sqrt{\alpha \beta} k_{0 y}} \frac{\partial v_{g}}{\partial k_{x}}\right|_{k_{0}}<0 .
$$

From the explicit expression for the derivative of the group velocity, one obtains

$$
\Omega=q v_{g}-i K_{q}|q| \frac{k_{0 y}}{k_{0 \lambda}} N_{0}^{1 / 2} \frac{\sqrt{2 k_{0 x}^{2}-k_{0 y}^{2}}}{k_{0}^{2}} .
$$

In this case, there is stabilization for $2 k_{0 x}^{2}-k_{0 y}^{2}<0$. Note that the instability conditions (31) as well as (35) are similar to the well known Lighthill criterion for modulational instability.

Following the same procedure as for zonal magnetic fields, the dispersion relation for magnetic streamers takes the form

$$
-i \Omega^{s t}=-K_{q}^{2} q^{2} \int \frac{k_{x}^{2} \lambda^{2}}{1+k^{2} \lambda^{2}} k_{y} \frac{\partial N_{0}}{\partial k_{y}} R(\Omega, q) d^{2} \mathbf{k} .
$$

The same arguments as before lead to the expression of the group velocity in the response function,

$$
v_{g}=\frac{\partial \omega_{k}}{\partial k_{y}}=\sqrt{\alpha \beta} \frac{1+k_{x}^{2} \lambda^{2}}{\left(1+k^{2} \lambda^{2}\right)^{3 / 2}} .
$$

Integrating (37) by parts yields

$$
\Omega=-K_{q}^{2} q^{2} \int k_{x}^{2} \lambda^{2} N_{0}^{k} \frac{\partial}{\partial k_{y}}\left(\frac{1}{\Omega-q v_{g}} \frac{k_{y}}{1+k^{2} \lambda^{2}}\right) d^{2} \mathbf{k} .
$$

In the limit $k \lambda \ll 1$, the group velocity (38) becomes a constant, $v_{g}=\sqrt{\alpha \beta}$, so that

$$
\frac{\partial}{\partial k_{y}}\left(\frac{1}{\Omega-q v_{g}} \frac{k_{y}}{1+k^{2} \lambda^{2}}\right)=\frac{1}{\Omega-q v_{g}},
$$

and therefore the integration of (39) with a monochromatic distribution can easily be performed and results in a quadratic equation for $\Omega$ with the solutions

$$
\Omega=\frac{1}{2} q\left[v_{g} \pm \sqrt{v_{g}^{2}-4 K_{q}^{2} q^{2} k_{0 x}^{2} \lambda^{2} N_{0}}\right] .
$$

Thus, the instability develops for $v_{g}=\sqrt{\alpha \beta}<2 K_{q} q k_{0 x} \lambda N_{0}^{1 / 2}$.

In the opposite limit $k \lambda \gg 1$, it is easier to calculate the derivative in (39) directly rather than replacing the term as a function of the group velocity before deriving. The solution for the resulting quadratic equation for $\Omega$ is in this case

$$
\Omega=\frac{1}{2} q\left[v_{g} \pm \sqrt{v_{g}^{2}-4 K_{q}^{2} q^{2} k_{0 x}^{2} N_{0} \frac{k_{0 x}^{2}-k_{0 y}^{2}}{k^{2} \lambda^{2}}}\right] .
$$

Then, there is instability for $v_{g}^{2}<4 K_{q}^{2} q^{2} k_{0 x}^{2} N_{0}\left(k_{0 x}^{2}\right.$ $\left.-k_{0 y}^{2}\right) /\left(k^{2} \lambda^{2}\right)$. First, note that the increment of the instability is weakened by a factor of $1 /\left(k^{2} \lambda^{2}\right) \ll 1$ compared to the long wavelength limit and, secondly, that if $k_{0 y}^{2}>k_{0 x}^{2}$, instability is not possible.

We have seen that the small scale wave packets are unstable with respect to large scale perturbations. As these wave packets are exited, they are accompanied by excitation of large scale fields. The mechanism studied here constitutes the kinetic and the hydrodynamic regimes of the same process, similar to the case of the electrostatic drift wave turbulence. $^{9}$

The last section has shown that large scale magnetic fields can be generated and strengthened through instabilities and therefore also subsist for a longer time scale. This is why we will turn our attention towards the long term dynamics in the next section.

\section{LONG TERM DYNAMICS OF LARGE SCALE FIELDS}

Having an equation for large scale fields and microturbulence and their mutual interaction, the wave kinetic equation (8), we now want to investigate the nonlinear evolution of zonal magnetic fields and magnetic streamers and look for long-lived coherent structures.

First, we concentrate on zonal magnetic fields. The explicit expressions for the velocities $\mathbf{v}_{B}$ and $\mathbf{v}_{T}$ in (10) are then $\mathbf{v}_{B}=e \lambda^{2} /(4 m) \partial_{x} B \hat{\mathbf{y}}$, and $\mathbf{v}_{T}=e \lambda^{2} /(4 m) \sqrt{\beta / \alpha} \partial_{x} B \hat{\mathbf{y}}$, respectively. Thus, the "flow velocity" defined in (11) takes the form

$$
\mathbf{v}_{f}=v_{f} \hat{\mathbf{y}}=\frac{e \lambda^{2}}{4 m} \frac{\partial}{\partial x}\left(\frac{1+2 k^{2} \lambda^{2}}{1+k^{2} \lambda^{2}} B-\frac{1}{\sqrt{1+k^{2} \lambda^{2}}} \sqrt{\frac{\beta}{\alpha}} T\right) \hat{\mathbf{y}} .
$$

It is convenient to introduce the vector

$$
B^{*} \equiv \frac{e \lambda^{2}}{4 m}\left[\frac{1+2 k^{2} \lambda^{2}}{1+k^{2} \lambda^{2}} B_{q}-\frac{1}{\sqrt{1+k^{2} \lambda^{2}}} \sqrt{\frac{\beta}{\alpha}} T_{q}\right] e^{i \mathbf{q} \cdot \mathbf{r}} .
$$

Note that in the long wavelength limit, $k \lambda \ll 1$, this vector coincides with $\bar{B}$ defined in (15). With this new notation, the flow velocity (43) can simply be expressed as $v_{f}=\partial_{x} B^{*}$.

We are interested in the evolution equation for the flow velocity $v_{f}$. From the above relations, the time derivative takes the form

$$
\frac{\partial v_{f}}{\partial t}=\frac{\partial}{\partial x} \frac{\partial B^{*}}{\partial t}=\frac{\partial}{\partial x}\left[\frac{e \lambda^{2}}{4 m} \frac{1+2 k^{2} \lambda^{2}}{1+k^{2} \lambda^{2}} \frac{\partial B_{q}}{\partial t} e^{i q x}\right],
$$

where we used the result from (5), in particular that the time derivative of the temperature vanishes. Our goal is to express the time derivative of the flow velocity in terms of large scale parameters. Taking into account that for zonal fields, $\mathbf{k} \cdot \mathbf{v}_{f}=i q k_{y} B^{*}$, and thus $\partial_{\mathbf{r}}\left(\mathbf{k} \cdot \mathbf{v}_{f}\right)=-q^{2} k_{y} B^{*} \hat{\mathbf{x}}$, and using these results in Eq. (14) yields the equation for the flow velocity

$$
\frac{\partial v_{f}}{\partial t}=-q^{2} D_{x x} \frac{\partial B^{*}}{\partial x}=-q^{2} D_{x x} v_{f},
$$

where we defined the coefficient 
$D_{x x} \equiv \frac{\beta}{16 \alpha}\left(\frac{e \lambda^{2}}{m}\right)^{2} \frac{1+2 k^{2} \lambda^{2}}{1+k^{2} \lambda^{2}} \int \frac{k_{y}^{2}}{1+k^{2} \lambda^{2}} k_{x} \frac{\partial N_{0}}{\partial k_{x}} R(\Omega, q) d^{2} \mathbf{k}$.

Up to now, we considered the resonant response with $\Omega \simeq q v_{g}$. With a nonlinear grow of zonal fields, we have to take into account higher order effects described by the nonresonant response, $\Omega \ll q v_{g}$, as well. To this end, we will decompose the wave spectrum into an equilibrium, a resonant and a nonresonant first order and a nonresonant second order perturbed part, $N_{k}=N_{0}+\widetilde{N}_{k}^{r}+\widetilde{N}_{k}^{(1)}+\tilde{N}_{k}^{(2)}$. The resonant solution $N_{k}^{r}$ is given by Eq. (14). For the nonresonant parts we use again the linearized wave kinetic equation (13), but this time with the assumption $\Omega \ll q v_{g}$ and using perturbation theory. For $\widetilde{N}_{k}^{(1)}$, this gives

$$
\tilde{N}_{k}^{(1)}=k_{y} v_{f}\left(\frac{\partial \omega}{\partial k_{x}}\right)^{-1} \frac{\partial N_{0}}{\partial k_{x}} .
$$

Inserting this result into the third term of Eq. (13) yields for the second order perturbation

$$
\tilde{N}_{k}^{(2)}=\left(k_{y} v_{f}\right)^{2}\left(\frac{\partial \omega}{\partial k_{x}}\right)^{-1} \frac{\partial}{\partial k_{x}}\left[\left(\frac{\partial \omega}{\partial k_{x}}\right)^{-1} \frac{\partial N_{0}}{\partial k_{x}}\right] .
$$

In order to use the above results, we need to express $\partial_{t} v_{f}$ as a function of the perturbed wave spectrum $\tilde{N}_{k}$. To this end, we use Eqs. (45), (5b), and (7). Then, (46) takes the form

$$
\begin{aligned}
\frac{\partial v_{f}}{\partial t}= & \frac{\partial}{\partial x}\left[\left(\frac{e \lambda^{2}}{m}\right)^{2} \frac{1+2 k^{2} \lambda^{2}}{1+k^{2} \lambda^{2}} \frac{q^{2} \lambda^{2}}{1+q^{2} \lambda^{2}}\right. \\
& \left.\times \frac{\beta}{4 \alpha} \int k_{x} k_{y} \frac{\tilde{N}_{k}}{1+k^{2} \lambda^{2}} d^{2} \mathbf{k}\right] .
\end{aligned}
$$

The different parts of the decomposition of the wave spectrum contribute as follows:

- For the resonant part, we have already seen that we get

$$
\frac{\partial v_{f}^{r}}{\partial t}=D_{x x} \frac{\partial^{2} v_{f}^{r}}{\partial x^{2}} .
$$

- For the nonresonant part we introduce the quantity

$$
\xi(k)=\frac{\beta}{4 \alpha}\left(\frac{e \lambda^{2}}{m}\right)^{2} \frac{1+2 k^{2} \lambda^{2}}{1+k^{2} \lambda^{2}} \frac{q^{2} \lambda^{2}}{1+q^{2} \lambda^{2}}
$$

and define

$$
u_{x} \equiv \xi(k) \int d^{2} \mathbf{k} \frac{k_{x} k_{y}^{2}}{1+k^{2} \lambda^{2}}\left(\frac{\partial \omega}{\partial k_{x}}\right)^{-1} \frac{\partial N_{0}}{\partial k_{x}},
$$

so that the first order nonresonant contribution is

$$
\frac{\partial v_{f}^{(1)}}{\partial t}=u_{x} \frac{\partial v_{f}^{(1)}}{\partial x} .
$$

- Finally, we introduce for the second order nonresonant perturbation the quantity

$$
b_{x} \equiv \xi(k) \int d^{2} \mathbf{k} \frac{k_{x} k_{y}^{3}}{1+k^{2} \lambda^{2}}\left(\frac{\partial \omega}{\partial k_{x}}\right)^{-1} \frac{\partial}{\partial k_{x}}\left[\left(\frac{\partial \omega}{\partial k_{x}}\right)^{-1} \frac{\partial N_{0}}{\partial k_{x}}\right] .
$$

Hence,

$$
\frac{\partial v_{f}^{(2)}}{\partial t}=b_{x} \frac{\partial}{\partial x}\left(v_{f}^{(2)}\right)^{2} .
$$

All we have to do now is to sum up (51), (53), and (55) in order to get the evolution equation for zonal fields,

$$
\frac{\partial}{\partial t} \frac{\partial}{\partial x} v_{f}=D_{x x} \frac{\partial^{3} v_{f}}{\partial x^{3}}+u_{x} \frac{\partial^{2} v_{f}}{\partial x^{2}}+b_{x} \frac{\partial^{2}}{\partial x^{2}} v_{f}^{2} .
$$

The same procedure can be applied to magnetic streamers. In this case, the explicit expressions for the velocities are $\mathbf{v}_{B}=-e \lambda^{2} /(4 m) \partial_{y} B \hat{\mathbf{x}}$ and $\mathbf{v}_{T}=-e \lambda^{2} /(4 m) \sqrt{\beta / \alpha} \partial_{y} B \hat{\mathbf{x}}$. Thus, the flow velocity defined in (11) takes the form

$$
\mathbf{v}_{f}=v_{f} \hat{\mathbf{x}}=-\frac{e \lambda^{2}}{4 m} \frac{\partial}{\partial y}\left(\frac{1+2 k^{2} \lambda^{2}}{1+k^{2} \lambda^{2}} B-\frac{1}{\sqrt{1+k^{2} \lambda}} \sqrt{\frac{\beta}{\alpha}} T\right) .
$$

As it can be easily seen, it is convenient to use the same vector $B^{*}$ as defined in (44). Then, the flow velocity can be expressed as $v_{f}=-\partial_{y} B^{*}$. The most significant difference to zonal magnetic fields can be found by comparing the evolution equations (5) and (6): While for ZF the time derivative of the temperature vanishes and therefore does not contribute in the expression for $\partial_{t} v_{f}$, it is nonzero for MS. This will result in an extra term in the evolution equation for the flow velocity.

For further discussions, we introduce another vector

$$
B^{\dagger} \equiv \frac{e \lambda^{2}}{4 m}\left[\frac{1}{\sqrt{1+k^{2} \lambda^{2}}} B_{q}-\frac{1+2 k^{2} \lambda^{2}}{1+k^{2} \lambda^{2}} \sqrt{\frac{\beta}{\alpha}} T_{q}\right] e^{i \mathbf{q} \cdot \mathbf{r}} .
$$

Note that in the limit $k \lambda \ll 1, B^{*}$ coincides with $B^{\dagger}$. From the evolution equations for magnetic streamers (6), we obtain the evolution equation for $B^{*}$,

$$
\begin{aligned}
\frac{\partial B^{*}}{\partial t}-i q \sqrt{\alpha \beta} B^{\dagger}= & \frac{1+2 k^{2} \lambda^{2}}{1+k^{2} \lambda^{2}}\left(\frac{e \lambda^{2}}{m}\right)^{2} \frac{q^{2} \lambda^{2}}{1+q^{2} \lambda^{2}} \\
& \times \int d^{2} \mathbf{k} k_{x} k_{y}\left|B_{k}\right|^{2} e^{i \mathbf{q} \cdot \mathbf{r}} .
\end{aligned}
$$

In order to replace the RHS of this equation, we use as before for ZF the relation (7) and the expression for the perturbed wave spectrum, Eq. (14), where in the case of MS, $\partial_{r}\left(\mathbf{k} \cdot \mathbf{v}_{f}\right)=k_{x} \partial_{y} v_{f} \hat{\mathbf{x}}$. So, the corresponding equation to (46) for the flow velocity in the resonant case is

$$
\frac{\partial v_{f}^{r}}{\partial t}=-D_{y y} \frac{\partial^{2} v_{f}^{r}}{\partial y^{2}}-i q \sqrt{\alpha \beta} \frac{\partial B^{\dagger}}{\partial y},
$$

where we defined the coefficient 
$D_{y y} \equiv \frac{\beta}{16 \alpha}\left(\frac{e \lambda^{2}}{m}\right)^{2} \frac{1+2 k^{2} \lambda^{2}}{1+k^{2} \lambda^{2}} \int \frac{k_{x}^{2}}{1+k^{2} \lambda^{2}} k_{y} \frac{\partial N_{0}}{\partial k_{y}} R(\Omega, q) d^{2} \mathbf{k}$.

Note that in the limit $k \lambda \ll 1,-\partial_{y} B^{\dagger}=v_{f}$, and there is, contrary to zonal fields, an oscillating part in the solution of $v_{f}$ from (60) with long wavelength $2 \pi / q$ and phase velocity $\sqrt{\alpha \beta}$.

As for the nonresonant case, $\Omega \ll q v_{g}$, the calculations are exactly the same as for zonal fields, and the first and second order perturbed wave spectra are then given by

$$
\tilde{N}_{k}^{(1)}=k_{x} v_{f}\left(\frac{\partial \omega}{\partial k_{y}}\right)^{-1} \frac{\partial N_{0}}{\partial k_{y}}
$$

and

$$
\tilde{N}_{k}^{(2)}=\left(k_{x} v_{f}\right)^{2}\left(\frac{\partial \omega}{\partial k_{y}}\right)^{-1} \frac{\partial}{\partial k_{y}}\left[\left(\frac{\partial \omega}{\partial k_{y}}\right)^{-1} \frac{\partial N_{0}}{\partial k_{y}}\right] .
$$

The expression of $\partial_{t} v_{f}$ as a function of the total perturbed wave spectrum $\widetilde{N}_{k}$ can be found recalling that $v_{f}=-\partial_{y} B^{*}$ and using Eqs. (59) and (7). The result is similar to the corresponding expression for zonal fields (50) with an additional term

$$
\begin{aligned}
\frac{\partial v_{f}}{\partial t}= & -\frac{\partial}{\partial y}\left[\left(\frac{e \lambda^{2}}{m}\right)^{2} \frac{1+2 k^{2} \lambda^{2}}{1+k^{2} \lambda^{2}} \frac{q^{2} \lambda^{2}}{1+q^{2} \lambda^{2}} \frac{\beta}{4 \alpha}\right. \\
& \left.\times \int k_{x} k_{y} \frac{\tilde{N}_{k}}{1+k^{2} \lambda^{2}} d^{2} \mathbf{k}+\sqrt{\alpha \beta} \frac{\partial B^{\dagger}}{\partial y}\right] .
\end{aligned}
$$

The contributions of the different parts of the decomposition of the wave spectrum contribute as follows:

- For the resonant part, the result is given by (60),

$$
\frac{\partial v_{f}^{r}}{\partial t}=-D_{y y} \frac{\partial^{2} v_{f}^{r}}{\partial y^{2}}-\sqrt{\alpha \beta} \frac{\partial^{2} B^{\dagger}}{\partial y^{2}} .
$$

- For the nonresonant part we use $\xi(k)$ defined above and the velocity

$$
u_{y} \equiv \xi(k) \int d^{2} \mathbf{k} \frac{k_{y} k_{x}^{2}}{1+k^{2} \lambda^{2}}\left(\frac{\partial \omega}{\partial k_{y}}\right)^{-1} \frac{\partial N_{0}}{\partial k_{x}}
$$

so that the first order nonresonant contribution is

$$
\frac{\partial v_{f}^{(1)}}{\partial t}=-u_{y} \frac{\partial v_{f}^{(1)}}{\partial y} .
$$

- Finally, we introduce for the second order nonresonant perturbation the quantity

$$
b_{y} \equiv \xi(k) \int d^{2} \mathbf{k} \frac{k_{y} k_{x}^{3}}{1+k^{2} \lambda^{2}}\left(\frac{\partial \omega}{\partial k_{y}}\right)^{-1} \frac{\partial}{\partial k_{y}}\left[\left(\frac{\partial \omega}{\partial k_{y}}\right)^{-1} \frac{\partial N_{0}}{\partial k_{y}}\right] .
$$

$$
\frac{\partial v_{f}^{(2)}}{\partial t}=-b_{y} \frac{\partial}{\partial y}\left(v_{f}^{(2)}\right)^{2}
$$

The sum of (65), (67), and (69) yields the evolution equation for magnetic streamers,

$$
\frac{\partial}{\partial t} \frac{\partial}{\partial y} v_{f}=-D_{y y} \frac{\partial^{3} v_{f}}{\partial y^{3}}-u_{y} \frac{\partial^{2} v_{f}}{\partial y^{2}}-b_{y} \frac{\partial^{2}}{\partial y^{2}} v_{f}^{2}-\sqrt{\alpha \beta} \frac{\partial^{3} B^{\dagger}}{\partial y^{3}} .
$$

We now have the evolution equations for zonal magnetic fields (56) and for magnetic streamers (70). As they are nonlinear, finding exact solutions is rather difficult. However, we can look for partial solutions in the form of stationary structures which are localized in space. The so found solutions will then describe nonlinear coherent structures.

\section{LOCALIZED SOLUTIONS}

As before, we first concentrate on zonal fields. We now look for stationary solutions of Eq. (56), propagating with constant velocity $u_{0 x}$ in the $x$ direction, $v_{f}\left(x-u_{0 x} t\right)$. Hence, after integrating Eq. (56) twice, one obtains

$$
\left(u_{x}+u_{0 x}\right) v_{f}+b_{x} v_{f}^{2}+D_{x x} \frac{\partial v_{f}}{\partial x}=C,
$$

where $C$ is the integration constant. We impose the boundary conditions corresponding to a solitary wave with different asymptotic values, i.e. a "switching" or "kink" soliton, which are $v_{y} \rightarrow v_{1}, v_{y}^{\prime} \rightarrow 0$ as $x \rightarrow-\infty$ and $v_{y} \rightarrow v_{2}, v_{y}^{\prime} \rightarrow 0$ as $x \rightarrow \infty$. The integration constant then takes the form $C=\left(u_{x}+u_{0 x}\right) v_{1}$ $+b_{x} v_{1}^{2}$ and we can express $v_{2}$ as a function of $v_{1}$ as $v_{2}=-v_{1}-\left(u_{x}+u_{0 x}\right) / b_{x}$. The simplest solution for Eq. (71) with these assumptions is given by ${ }^{35}$

$$
v_{f}=\frac{1}{2}\left\{v_{1}+v_{2}+\left(v_{1}-v_{2}\right) \tanh \left[\frac{b_{x}\left(v_{1}-v_{2}\right)}{2 D_{x x}} x\right]\right\} .
$$

This solution describes the transient region between two different values of the flow $v_{1}$ to $v_{2}$. We note that it is different from the stationary vortex solution found earlier. ${ }^{21,22}$ The cooperative effects of the wave motion, steepening and instability give the possibility of forming stationary or moving kink solitons in between the surfaces of two different flow velocities. We would expect the effect of modifying the anomalous electron transport properties within the plasma as stated earlier. In a polar geometry, zonal fields are elongated along the $\theta$ direction. They are known to inhibit anomalous transport in the radial direction by shearing small scale turbulence. ${ }^{1}$ However, the coherent structures defined by Eq. (72) travelling along the radial direction with the velocity $u_{0 x}$, take trapped particles with them and hence can even attribute to radial electron transport.

The case of magnetic streamers is similar to the above case with one difference, which is the additional term in the streamer flow velocity evolution equation (70). We look for stationary solutions of the form $v_{f}\left(y-u_{0 y} t\right)$ and replace the time with a space derivative. Integrating Eq. (70) twice yields

And thus, 


$$
\left(u_{y}-u_{0 y}\right) v_{f}+b_{y} v_{f}^{2}+D_{y y} \frac{\partial v_{f}}{\partial y}+\sqrt{\alpha \beta} \frac{\partial B^{\dagger}}{\partial y}=C .
$$

As already denoted earlier, there is an interesting limit $k \lambda \ll 1$, for which $\partial_{y} B^{\dagger} \rightarrow v_{f}$. In this case, the latter equation is simplified to

$$
\left(u_{y}+\left(\sqrt{\alpha \beta}-u_{0 y}\right)\right) v_{f}+b_{y} v_{f}^{2}+D_{y y} \frac{\partial v_{f}}{\partial y}=C .
$$

The result is very similar to zonal fields (71), $x$ and $y$ being permutated. But there seems to be a Doppler effect due to the already observed large scale oscillations with phase velocity $\sqrt{\alpha \beta}$, so that the localized solution does not see the translation velocity $u_{0 y}$, but the difference between translation velocity and the phase velocity.

\section{CONCLUSIONS}

The properties of large scale fields, both magnetic streamers and zonal magnetic fields, have been investigated using a self-consistent model for magnetic electron drift wave turbulence. The small scale turbulence evolves in a medium of slowly changing variables, large scale fields, and is modulated by them. The kinetic and hydrodynamic instability of these modulations have been studied and in the hydrodynamic case, a criterion similar to the Lighthill criterion for the case of modulational instability has been found for zonal fields. Once it was shown that large scale fields can be unstable and be strengthened, their nonlinear long-term evolution could be studied. We were able to stress out that both zonal magnetic fields and magnetic streamers admit the formation of stationary coherent structures in the transition layer between surfaces of different flow velocities, modifying the turbulent electron transport properties. In the case of magnetic streamers, the translation velocity was found to be Doppler shifted, but in both cases they found switching solitons were propagating perpendicularly to the direction of elongation of the large scale magnetic structures.

\section{ACKNOWLEDGMENTS}

M.J. would like to acknowledge the hospitality of the Department of Astronomy and Space Physics during his stay at Uppsala University as well as the support and openmindedness of Professor Laurent Villard and the École Polytechnique Fédérale in Lausanne, Switzerland, in the frame of bilateral scientific exchange.
${ }^{1}$ P. H. Diamond, S.-I. Itoh, K. Itoh, and T. Hahm, Plasma Phys. Controlled Fusion 47, 35 (2005).

${ }^{2}$ P. H. Diamond and Y.-B. Kim, Phys. Fluids A 3, 1626 (1991).

${ }^{3}$ M. A. Malkov and P. H. Diamond, Phys. Plasmas 9, 3996 (2001).

${ }^{4}$ A. Vedenov, A. Gordeev, and L. Rudakov, Plasma Phys. 9, 719 (1967).

${ }^{5}$ A. Smolyakov and P. Diamond, Phys. Plasmas 6, 4410 (1999).

${ }^{6}$ P. H. Diamond, M. N. Rosenbluth, F. L. Hinton, M. Malkov, J. Fleischer, and A. Smolyakov, in Plasma Physics and Controlled Nuclear Fusion Research, 17th IAEA Fusion Energy Conference, Yokohama, Japan, 1998 (International Atomics Energy Agency, Vienna, 1998).

${ }^{7}$ P. H. Diamond, M. N. Rosenbluth, F. L. Hinton, M. Malkov, J. Fleischer, and A. Smolyakov, in Plasma Physics and Controlled Nuclear Fusion Research, 18th IAEA Fusion Energy Conference, Sorrento, Italy, 2000 (International Atomics Energy Agency, Vienna, 2000).

${ }^{8}$ M. A. Malkov, P. H. Diamond, and A. Smolyakov, Phys. Plasmas 8, 1553 (2001).

${ }^{9}$ A. Smolyakov, P. H. Diamond, and V. Shevchenko, Phys. Plasmas 7, 5 (2000).

${ }^{10}$ K. Itoh, K. Hallatschek, S.-I. Itoh, P. H. Diamond, and S. Toda, Phys. Plasmas 12, 062303 (2005).

${ }^{11}$ A. Smolyakov, P. Diamond, and Y. Kishimoto, Phys. Plasmas 9, 3826 (2002).

${ }^{12}$ W. P. Wilkinson, Planet. Space Sci. 51, 629 (2003).

${ }^{13}$ A. Das and P. H. Diamond, Phys. Plasmas 7, 170 (2000).

${ }^{14}$ D. A. Tidman and R. A. Shanny, Phys. Fluids 17, 1207 (1974).

${ }^{15}$ A. Z. Dolginov and V. A. Urpin, Zh. Eksp. Teor. Fiz. 77, 1921 (1979).

${ }^{16}$ R. D. Jones, Phys. Rev. Lett. 51, 1269 (1983).

${ }^{17}$ P. Amendt, H. U. Rahman, and M. Strauss, Phys. Rev. Lett. 53, 1226 (1984).

${ }^{18}$ M. Yu and L. Stenflo, Phys. Fluids 28, 3447 (1985).

${ }^{19}$ L. Stenflo and M. Yu, Phys. Fluids 29, 2335 (1986).

${ }^{20}$ M. Y. Yu and X. Chijin, Phys. Fluids 30, 3631 (1987).

${ }^{21}$ J. Nycander, V. Pavlenko, and L. Stenflo, Phys. Fluids 30, 1367 (1987).

${ }^{22}$ J. Nycander and V. P. Pavlenko, Phys. Fluids B 3, 1386 (1991).

${ }^{23}$ V. P. Pavlenko and L. Uby, Phys. Fluids B 5, 1980 (1993).

${ }^{24}$ V. P. Pavlenko and L. Uby, Phys. Plasmas 1, 2140 (1994).

${ }^{25}$ Zh. N. Andrushchenko and V. P. Pavlenko, Phys. Plasmas 11, 1402 (2004).

${ }^{26}$ Zh. N. Andrushchenko, M. Jucker, and V. P. Pavlenko, "Self-consistent model of electron drift mode turbulence" (in preparation).

${ }^{27}$ J. C. Charney, J. Atmos. Sci. 28, 1087 (1971).

${ }^{28}$ A. Das, R. Singh, P. Kaw, and S. Champeaux, Phys. Plasmas 9, 2609 (2002).

${ }^{29}$ C. Holland and P. Diamond, Phys. Plasmas 9, 3857 (2002).

${ }^{30}$ K. Subramanian, Phys. Rev. Lett. 90, 245003 (2003).

${ }^{31}$ L. Rudakov, Phys. Plasmas 12, 042111 (2005).

${ }^{32}$ V. Lebedev, P. Diamond, V. Shapiro, and G. Soloviev, Phys. Plasmas 9, 719 (1995).

${ }^{33}$ R. Z. Sagdeev and A. A. Galeev, Nonlinear Plasma Theory (Benjamin, New York, 1969).

${ }^{34}$ A. A. Vedenov and L. I. Rudakov, Sov. Phys. Dokl. 9, 1073 (1965).

${ }^{35}$ A. Smolyakov, P. H. Diamond, and M. A. Malkov, Phys. Rev. Lett. 84, 491 (2000). 